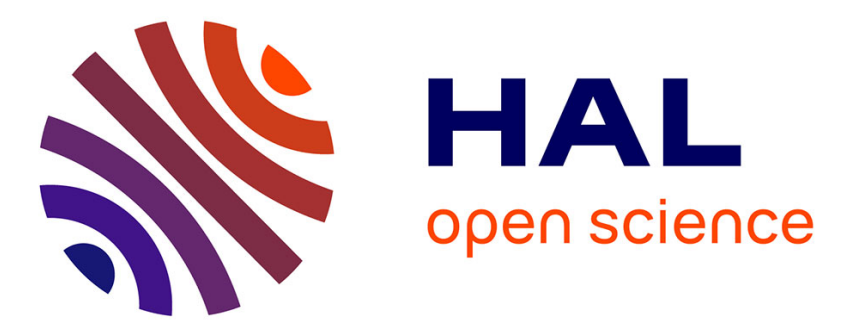

\title{
Flame-vortex interaction: Effect of residence time and formulation of a new efficiency function
}

\author{
F. Thiesset, G. Maurice, F. Halter, Nicolas Mazellier, C. Chauveau, I. Gökalp
}

\section{To cite this version:}

F. Thiesset, G. Maurice, F. Halter, Nicolas Mazellier, C. Chauveau, et al.. Flame-vortex interaction: Effect of residence time and formulation of a new efficiency function. Proceedings of the Combustion Institute, 2017, 36 (2), pp.1843 - 1851. 10.1016/j.proci.2016.06.172 . hal-01660275

\section{HAL Id: hal-01660275 https://hal.science/hal-01660275}

Submitted on 28 Mar 2019

HAL is a multi-disciplinary open access archive for the deposit and dissemination of scientific research documents, whether they are published or not. The documents may come from teaching and research institutions in France or abroad, or from public or private research centers.
L'archive ouverte pluridisciplinaire HAL, est destinée au dépôt et à la diffusion de documents scientifiques de niveau recherche, publiés ou non, émanant des établissements d'enseignement et de recherche français ou étrangers, des laboratoires publics ou privés. 


\title{
Flame-vortex interaction : effect of residence time and formulation of a new efficiency function
}

\author{
F. Thiesset ${ }^{\mathrm{a}}$, G. Maurice ${ }^{\mathrm{a}, \mathrm{b}}$, F. Halter ${ }^{* a}$, N. Mazellier ${ }^{\mathrm{b}}$, C. Chauveau $^{\mathrm{a}}$, I. Gökalp ${ }^{\mathrm{a}}$ \\ ${ }^{a}$ CNRS ICARE, Avenue de la Recherche Scientifique, 45072 Orléans Cedex 2 France \\ ${ }^{b}$ University of Orléans, INSA de Bourges, PRISME, EA 4229, 45072 Orléans, France
}

\begin{abstract}
In this study, a combined experimental and numerical investigation of a toroidal vortex interacting with a stagnation premixed flame is carried out with the aim of quantifying the ability of such a vortex to stretch the flame. By scrutinizing the literature, it was found that, although inferred from exactly similar numerical simulations, existing parametric expressions for the efficiency function (the ratio of the flame stretch to vortex strain) do not agree in the way the latter should behave when the ratio of the vortex rotational velocity $U_{\theta}$ to the laminar flame speed $S_{L}$ is increased. These expressions also appear to be unequally accurate when compared to experimental data and do not feature the non monotonic evolution of the efficiency function with $U_{\theta} / S_{L}$ which is observed in both experimental data and numerical simulations of a 'isothermal' propagating interface. In addition, whilst previous studies have focused only on the impact of $U_{\theta} / S_{L}$ and $R_{v} / \delta_{L}\left(R_{v}\right.$ being the vortex typical size and $\delta_{L}$ the laminar flame thickness) our study reveals the importance of other parameters, the most important of which being the residence time of the vortex associated with its convection velocity. These results yield a new formulation for the efficiency function which compares favourably well with experimental data.
\end{abstract}

Keywords: Flame vortex interactions, Flame stretch, Vortex strain, residence time

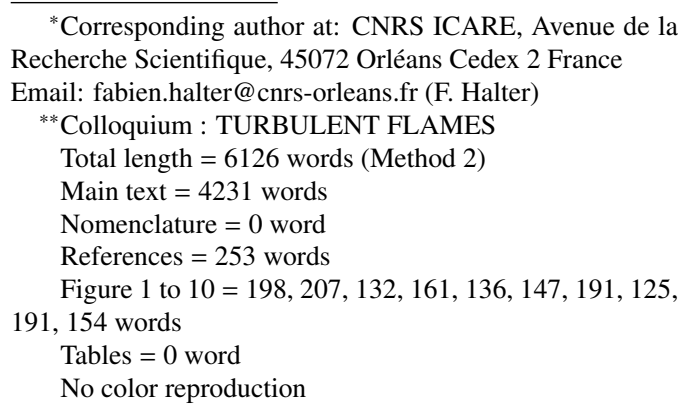




\section{Introduction}

Understanding and predicting the different mechanisms at play in turbulent premixed flames is a tremendously difficult challenge. The main reason is that there is still a lack of knowledge of the turbulent flow structure which features a large variety of turbulent scales. A given eddy thus experiences many different processes induced by turbulent scales of different sizes, such as vortex stretching and sweeping, diffusion by viscosity, these effects being particularly arduous to model. In addition, when reacting flows are concerned, the flame does not act as a passive scalar because of its propagative character and the inherent heat release that locally modifies the fluid physical properties. The high local flame curvature and strain, also impact its local consumption or displacement speed in a way which remains poorly understood.

There is thus a need for fundamental investigations of the interactions between the fluid motion and a flame in simplified and well controlled situations. One of these is the case of a flame interacting with a single vortex dipole (see the review by Renard et al. [1]). Pioneer studies of Flame-Vortex Interactions (hereafter abbreviated by FVI) emerged in the 90's with notably Poinsot et al. [2], Roberts and Driscoll [3], Wu and Driscoll [4], Roberts et al. [5], Lee and Santavicca [6] and more recently with Renard et al. [1], Colin et al. [7], Charlette et al. [8], Bougrine et al. [9].

Although some effects such as vortex stretching, sweeping, tilting are not present, FVI are expected to mimic, at least partly, the processes at play in real turbulent flames. The aforementioned investigations on FVI have led notably to the construction of the socalled spectral diagrams which allows to identify the conditions needed for a vortex to stretch the flame, to create pockets of fresh gas or to locally quench the flame. In addition, these results yielded expression of efficiency functions, i.e. the transfer function between vortex strain and flame stretch. In this prospect, Colin et al. [7], Charlette et al. [8] have focused on the effect of vortex size $R_{v}$ relative to the flame thickness $\delta_{L}$ and vortex rotational velocity $U_{\theta}$ relative to the flame speed $S_{L}$. More recently, the effect of Lewis number has been incorporated by Bougrine et al. [9]. These efficiency functions are extremely valuable as they are 105 widely used in LES of turbulent premixed combustion in order to model the sub-grid scale wrinkling factor 106 [7. 9].

The aim of the present study is to explore one par- 108 ticular aspect of the interaction between the flow mo- 109 tion and a flame, which we referred to as the strain- 110 sweeping competition (see for instance the review by 111 Driscoll [10|). This competition can be conceptu- 112 ally described in terms of time-scales. Based on phe- 113 nomenological arguments [11], the strain-based time 114 scale $\tau_{s}$ of a scale $r$ with characteristic velocity $u_{r}$ is 115 $\tau_{s} \propto r / u_{r} \propto r^{2 / 3}$. This time-scale is generally re- 116 ferred to as the eddy turn-over time. Previous studies 117 devoted to FVI investigations [2] indicate that the 118 smaller this time scale, the larger is the flame stretch. On the other hand, Tennekes [12] suggested that another relevant time scale in a turbulent flow relates to the sweeping effect by energy-containing eddies. He pointed out that a given scale of size $r$ is convected by the large scales, i.e. with characteristic velocity of the order of $u^{\prime}$, the root-mean-square of the velocity fluctuations. The sweeping time scale as called by Tennekes [12] thus writes $\tau_{c} \propto r / u^{\prime}$. This has been verified experimentally by e.g. Poulain et al. [13]. It is worth stressing that these two phenomenology both lead to the same prediction for the scaling exponent of the energy spectra and are therefore undistinguishable in spectral space. In the field of combustion the sweeping time scale is somehow related to the residence time [10] and basically describes the duration of the interaction of a vortex located in the vicinity of the flame. As far as the sweeping (or residence) time scale is concerned, FVI [3 4] corroborates the intuitive statement that the smaller this time scale, the smaller the flame stretch since the vortex spends less time in the vicinity of the flame for rolling it up. In turbulent flames, there is thus a competition between turbulent strain and turbulent convection, the latter phenomenon acts in decreasing the flame stretch whereas the former has the opposite effect. It is thus worth investigating these effects independently in order to give further insight into their respective influence on the flame. Further, a more complete expression for the efficiency function which accounts for both strain and residence time effects could be derived and used in LES.

In the present study, a new experimental set-up was designed in the goal of quantifying the degree of the interactions between a vortex dipole and a stagnation premixed flame. Some simple numerical simulations based on the 'isothermal' G-equation, have been further carried out and validated against experimental data. Such simulations allow to assess the effect of the convection velocity and rotational velocity independently. Finally, the respective effect of these two phenomena on flame stretch are separately quantified, incorporated into a new formulation for the efficiency function, and compared to experimental data.

\section{Experimental apparatus}

Investigations are carried out in a single jet stagnation flame configuration which is a modified version of that used by Bouvet et al. [14]. A schematic of the burner is provided in Fig. 1. A laminar strained flame is stabilized against a 4-mm-thick stainless steel plate. The stagnation plate is attached to an alumina foam plug selected for its insulating properties. The fuel and oxidizer are introduced through the side of the burner. A so-called 'particle diffuser cone' filled with $6 \mathrm{~mm}$ glass beads is used to ensure a homogeneous mixture in the nozzle plenum. The reactive mixture then flows into the burner plenum through a $5 \mathrm{~mm}$ thick aluminium grid. It is finally accelerated in the 


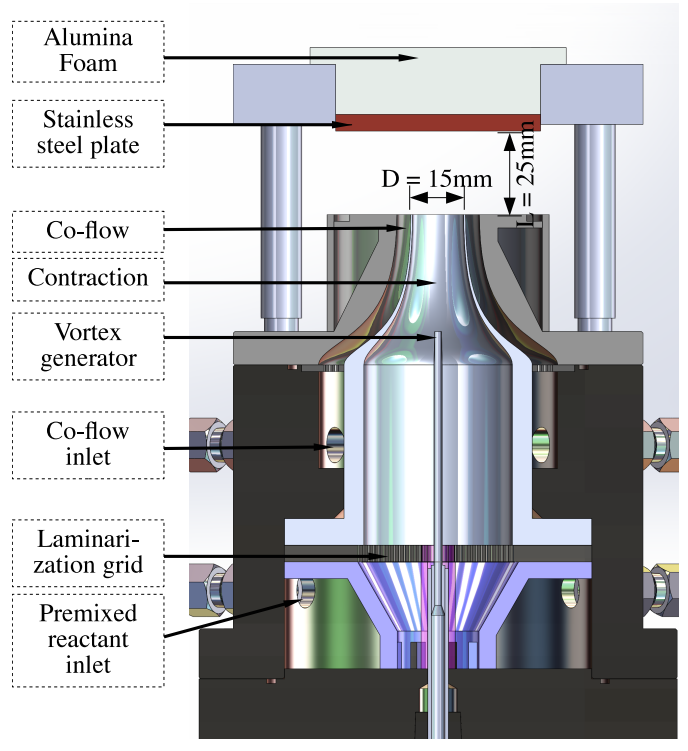

Figure 1: Schematic of the experimental setup

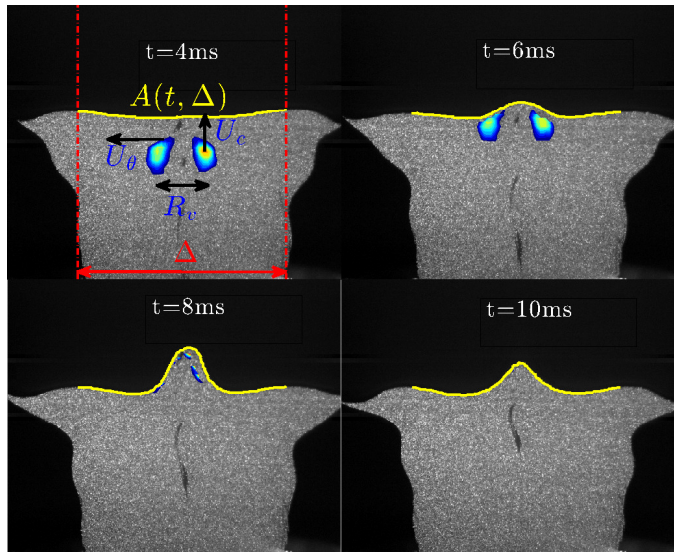

Figure 2: Time sequence of Mie scattering images of a typical FVI $\left(U_{\theta} / S_{L}=1.43\right)$. The flame contours and vorticity field are superimposed. The top of each image has been cropped to show only the first $20 \mathrm{~mm}$. The line corresponds to the flame contours with area $A(t, \Delta)$ estimated over a domain of width $\Delta . U_{\theta}, U_{c}$ are the vortex rotational and convection respectively, whilst $R_{v}$ is the vortex core-to-core distance.

laminar flame speed and thickness have been evaluated using the GRI-mech 3.0 mechanism together with the stagnation flame module of the CHEMKIN Pro software. The temperature of the wall, measured by Bouvet et al. [14], was set to $800 \mathrm{~K}$. It was found that $S_{L}=40.3,36.5,30.6 \mathrm{~cm} \cdot \mathrm{s}^{-1}$ and $\delta_{L}=433,463$, $525 \mu \mathrm{m}$ respectively for $\phi=1,0.9$ and 0.8 . The strain rate was respectively 90,86 and $77 \mathrm{~s}^{-1}$ for $\phi=1,0.9$ and 0.8 .

The flame front is tracked by means of Mie scattering laser tomography. Seeding of the flow is made by silicon oil droplets supplied by an atomizer. Typical size of droplets is about $1 \mu \mathrm{m}$. It was checked that the flame location was the same when the seeding was turned off suggesting that the laminar flame speed was not altered by the addition of silicon droplets in the flow. Then, use is made of a continuous Coherent Verdi G20 Laser which delivers up to $20 \mathrm{~W}$ at $532 \mathrm{~nm}$. The light scattered by the droplets is then captured by a Phantom V1210 camera, equipped with a $105 \mathrm{~mm}$ F2.8 lens, working at an acquisition rate of $23005 \mathrm{~Hz}$ with a field of view of $704 \times 640$ pixels $^{2}$ and the resolution was $38 \mu \mathrm{m} / \mathrm{px}$.

The flame contour is then extracted as follows. Firstly, a contrast-limited adaptive histogram equalization (CLAHE) is applied to the original images in order to optimize the contrast in the images. Then, to limit the pixelization associated with the CLAHE, images are filtered using a Gaussian filter of size equal to 4 times the spatial resolution. For the binarizing procedure, we use a standard threshold-based technique. More precisely, the histogram of the gray scale is calculated. The latter reveals two distinct peaks corresponding to the fresh and burned gas respectively. The threshold value for discriminating the flame con- 
tour is set as the average value between the gray scale of these two peaks. This yields estimations for the progress variable, noted $c$, which is by definition 0 and 1 in the unburned and burned gas respectively. The velocity field within the unburned mixture is estimated by classical 2D-2C Particle Image Velocimetry (PIV). For this purpose, the Matlab subroutines of Thielicke and Stamhuis [15] were used. A time sequence of Mie scattering images at four distinct instants is shown in Fig. 2 The vorticity field and flame contours are superimposed. The time $t_{0}=0$ was set arbitrarily as the time $t$ where the vortex center was $2.5 \mathrm{~mm}$ downstream the burner outlet. One observes that at a time $t=4 \mathrm{~ms}$, the flame is rather flat suggesting that the vortex generator is sufficiently far from the burner outlet for not creating a wake. As the vortex is convected $(t=6 \mathrm{~ms}$ and $8 \mathrm{~ms}$ ), the flame is increasingly stretched. Its area then reaches a maximum before decreasing $(\mathrm{t}=10 \mathrm{~ms})$ while the flame goes back to its original position.

The vortex parameters, i.e. the circumferential velocity $U_{\theta}$, the convection velocity $U_{c}$ and the coreto-core distance $R_{v}$, have been inferred from PIV by fitting the velocity field calculated with an Oseen vortex. Our experimental set-up allows to cover the range $0.5 \lesssim U_{\theta} / S_{L} \lesssim 2.5$ whereas $R_{v} / \delta_{L}$ slightly varies around 6.5. Our database thus lies between the no-effect limit and the quenching limit assessed by Roberts et al. [5].

\section{Experimental results}

\subsection{Domain size effects} sociated with the interaction with a vortex. Given the 258 vortex rotational velocity $U_{\theta}$ and the distance between 259 vortex cores $R_{v}$ (see Fig. 2), the vortex strain is gener- 260 ally estimated as $U_{\theta} / R_{v}[7,-9]$. On the other hand, the 261 flame stretch is evaluated as

$$
K(t, \Delta)=\frac{1}{A(t, \Delta)} \frac{\partial A(t, \Delta)}{\partial t}
$$

where, thanks to axisymmetry, $A(t, \Delta)=265$ $\int y(s) \sqrt{x^{\prime 2}+y^{\prime 2}} d s$ is the flame area at a time $t 266$ evaluated over a domain of width $\Delta$ (see Fig. 2). 267 $S$ is the curvilinear parameter, $y$ and $x$ are the 268 flame contour spatial coordinates and the prime 269 denotes derivatives with respect to $s$. Then the 270 efficiency function is defined as in [7.-9], viz. 271 $C(\Delta)=K_{\max }(\Delta) /\left(U_{\theta} / R_{v}\right)$, where $K_{\max }(\Delta)$ is the 272 maximum value of $K(t, \Delta)$. The appearance of 273 $\Delta$ in the efficiency function is new. In previous 274 numerical studies [7. 9] a given value for $\Delta \approx 6 R_{v} 275$ corresponding to the size of the simulation domain 276 was chosen. However, it appears straightforward 277 that $K_{\max }$ depends on $\Delta$. Indeed, because the portion 278 of flame interacting with the vortex is constant (i.e. 279 there exists a $\Delta$ above which $\partial A / \partial t$ is independent of 280 $\Delta)$, we expect $K_{\max }$ to decrease with $\Delta^{2}$ since $A(t, \Delta) \quad 281$ monotonically increases with $\Delta^{2}$. Figure 3 presents 282

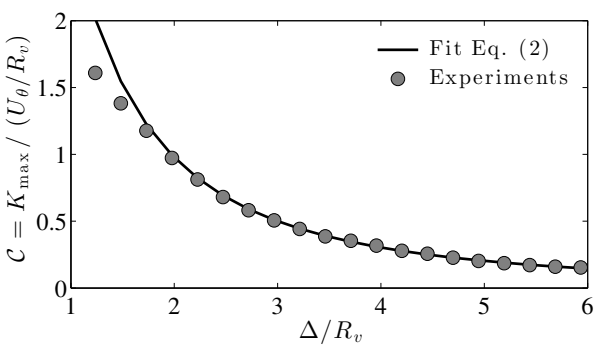

Figure 3: Evolution of $K_{\max }$ with $\Delta$ for $U_{\theta} / S_{L}=1.43$. Symbols represent experimental data whilst the line corresponds to the fit using Eq. 2).

the evolution of $K_{\max }$ with respect to $\Delta$. It clearly appears that $K_{\max }$ rapidly decreases with respect to $\Delta$ and for $\Delta$ sufficiently large (i.e. for $\Delta$ larger to a certain $\Delta_{i}$ ), it is found that $K_{\max }$ follows the relation

$$
K_{\max }=K_{\max }^{0}\left[\frac{\Delta_{i}-\Delta_{0}}{\Delta-\Delta_{0}}\right]^{2} .
$$

In Eq. (2), $\Delta_{i}$ represents the domain width above which $\partial A / \partial t$ is constant and $\Delta_{0}$ is interpreted as a virtual origin, i.e. $K_{\max }^{-1} \rightarrow 0$ when $\Delta \rightarrow \Delta_{0}$. From our experimental database, it was found that $\Delta_{i} / R_{v}$ and $\Delta_{0} / R_{v}$ were constant and are equal to $2.5 \pm 0.05$ and $-0.5 \pm 0.1$ respectively.

Figure 3 emphasizes that the values for the efficiency function that were previously provided notably by Colin et al. [7], Charlette et al. [8], Bougrine et al. [9], were inferred for a given value of $\Delta / R_{v}$ whereas they should depend on $\Delta$. In other words, if they had chosen a different value for the simulation domain, they would have obtained different values. Moreover, the no-effect limit assessed by Poinsot et al. [2] which "corresponds to vortices which induce a maximum modification of the total reaction rate of about 5 percent", should also depend on $\Delta$

\subsection{Impact of vortex intensity}

We now turn our attention to the effect of the vortex strength on the flame stretch. Figure 4 depicts the evolution of $C^{0}=K_{\max }^{0} /\left(U_{\theta} / R_{v}\right)$ as a function $U_{\theta} / S_{L}$ (hereafter the superscript ${ }^{0}$ on $C$ will be removed for the sake of simplicity). Experimental results are also compared to the predictions provided by Colin et al. [7], Charlette et al. [8], Bougrine et al. [9] which are respectively noted $C_{c o}, C_{c h}$ and $C_{b}$. Their respective analytical expressions are not recalled here but the reader can refer to [7. 9] for more details.

Experimental uncertainties have been estimated as follows. The precision of the subpixel interpolation of the PIV algorithm is generally about 0.05 pixel. The uncertainty on the velocity field is therefore constant and equals to about $0.04 \mathrm{~m} . \mathrm{s}^{-1}$ provided the resolution and sampling frequency of our images. The error on the estimation of $R_{v}$ provided by fitting experimental 


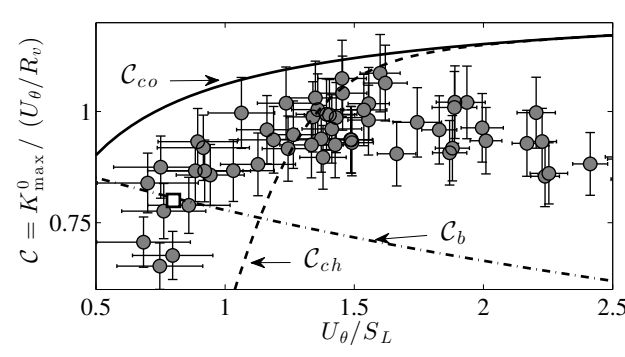

Figure 4: Efficiency function $C=K_{\max }^{0} /\left(U_{\theta} / R_{v}\right)$ as a function of $U_{\theta} / S_{L}$. The lines labbeled $C_{c o}, C_{c h}, C_{b}$ correspond respectively to the parametric expressions provided by Colin et al. [7], Charlette et al. [8], Bougrine et al. [9], corrected using Eq. 22 to obtain $K_{\max }^{0}$. The circle symbols correspond to the present measurements whilst the square symbol is taken from Bougrine et al. [9]

data with an Oseen vortex was generally of about $4 \%$

The uncertainty in the determination of $K_{\max }$ was supposed to be negligible by comparison with the errors on both $U_{\theta}$ and $R_{v}$ since $A$ is readily measurable.

A careful analysis of Fig. 4 first reveals that, al- 330 though rather limited, some departures between ex- 331 perimental data and the predictions of either [7- [9] can be observed. By comparison with experiments, ${ }^{33}$ the efficiency function of Colin et al. [7] appears to ${ }^{334}$ be the more appropriate. These differences might be ${ }^{335}$ explained by several parameters. First, it is worth ${ }^{336}$ recalling that our configuration is axisymetric whilst ${ }^{337}$ DNS of [7- 9] are 2D (planar). Secondly, in [7- [9], ${ }^{338}$ the flame stretch is estimated from the heat release ${ }^{339}$ $Q$, i.e. $K=Q^{-1} d Q / d t$ which implicitly suggests ${ }^{340}$ that Refs. [7- 7 ] considered that the flame consump- ${ }^{341}$ tion speed was unaltered by the flame stretch. Such ${ }^{342}$ an hypothesis is consistent with LES models based ${ }^{343}$ on the flame density concept for which the heat re- ${ }^{344}$ lease or fuel consumption is calculated through the 345 laminar flame speed multiplied by the flame surface 346 density. This assumption is however not consistent 347 with LES that employs skeletal or analytical chem- 348 istry which explicitly accounts for the effect of stretch 349 on the flame consumption speed.

Fig. 4 also suggests that though based on exactly 351 similar simulations, existing parametric expressions 352 do not agree in the way $C$ should behave with re- 353 spect to $U_{\theta} / S_{L}$. Indeed, Colin et al. 17], Charlette 354 et al. [8] both predict an increasing tendency of $C_{355}$ with respect to $U_{\theta} / S_{L}$ whereas $C_{b}$ leads to the op- 356 posite trend. Although slightly scattered, our exper- 357 imental data further suggest that the evolution of $C$ is 358 non monotonic, i.e. $C$ first increases before decreasing 359 slightly for $U_{\theta} / S_{L}$ larger than about 1.5 . The decreas- 360 ing tendency of $C$ was also observed in the DNS of ${ }_{361}$ Bougrine et al. [9] when the vortex strength was en- 362 hanced from $U_{\theta} / S_{L}=0.8$ to 8 (note that there is a nice 363 agreement between our experiments and the DNS data 364 of Bougrine et al. [9] for $U_{\theta} / S_{L}=0.8, R_{v} / \delta_{L}=5$ ). ${ }_{365}$ This observation can be readily explained by recalling 366

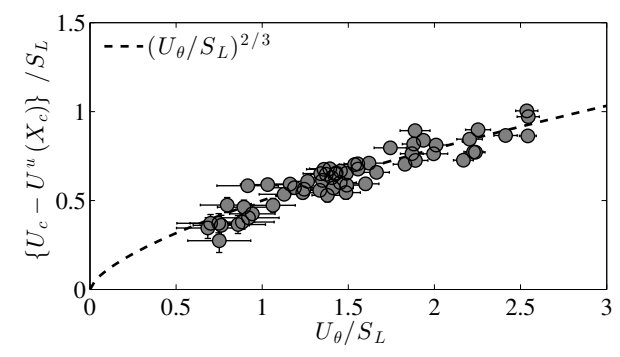

Figure 5: Convection velocity $U_{c}-U^{u}\left(X_{c}\right)$, where $U^{u}\left(X_{c}\right)$ is the streamwise velocity of the unperturbed flow at the vortex center location $X_{c}$, as a function of $U_{\theta}$. The dotted line is given by $0.5\left(U_{\theta} / S_{L}\right)^{2 / 3}$.

that an intense vortex will create high local curvatures which act in decreasing the total stretch of the flame. In other words, increasing the vortex strength can be less efficient since it leads to too high curvatures that globally reduces the flame stretch.

Roberts and Driscoll [3] were first to realize that the flame stretch is also driven by the convection velocity $U_{c}$ of the vortex dipole. More precisely, they suggested that for a given $U_{\theta}$, increasing $U_{c}$ yields a smaller flame stretch because the residence time of the vortex in the vicinity of the flame decreases. This intuitive statement was further confirmed by $\mathrm{Wu}$ and Driscoll [4] on the basis of numerical simulations of a propagating surface. There is thus a need for incorporating these two opposed effects (convection vs rotational velocity) into a more complete expression of the efficiency functions. However, in our experiments, it was observed that increasing $U_{\theta}$ irremediably led to a higher convection velocity consistently with analytical studies (see [1] and references therein). It was found experimentally (Fig. 5) that the convection velocity $U_{c}-U^{u}\left(X_{c}\right)\left(U^{u}\right.$ the streamwize velocity experienced by the vortex located at $X_{c}$ ) scales as $U_{\theta}^{2 / 3}$. Therefore, it is not possible from experiments to assess independently the respective influence of $U_{\theta}$ and $U_{c}$.

Consequently, following e.g. Wu and Driscoll [4 or Lee and Santavicca [6], we decided to perform simplified numerical simulations of the same burner in the goal of studying the effect of $U_{c}$ and $U_{\theta}$ independently. These simulations have been widely used in the past mainly because they are extremely low-cost in terms of computational resources. Indeed, they consider the flame as a 'passive' propagating (thermally neutral) interface, which tremendously reduces the problem complexity. Such simulations neglect the heat release and therefore many physical mechanisms are not taken into account. First, the higher viscosity due to high temperature in the burnt gas, resulting in a larger dissipation rate is not accounted for. Baroclinic effects as evidenced by [5] are also neglected. However, with this limitations in mind, one aspect of the present work is to investigate in detail how real- 


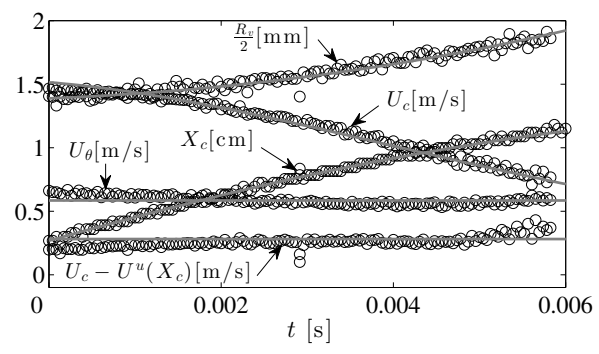

Figure 6: Time evolution of the vortex parameters for a given case in the database. The vortex core-to-core distance $R_{v}$, the convection velocity $U_{c}$ and $U_{c}-U^{u}\left(X_{c}\right)$, the vortex center streamwize position $X_{c}$, and the rotational velocity (black) are represented as a function of time. Symbols are experimental data whilst lines stand for the simulation.

istic these (widely used) simulations could be when compared to experiments. In other words, handling such a comparison helps in giving further insights into the importance of heat release and associated physical mechanism during a FVI.

\section{Simulations of a vortex interacting with a propagating interface}

\subsection{Implementation and validation}

Present numerical simulations consider the flame as a two-dimensional (axisymmetric) propagating interface convected by the fluid motion $\boldsymbol{U}$ while advancing at the laminar flame speed $S_{L}$. The kinematic relationship between the flame and the flow field is then given by the G-equation which writes [4, 6]

$$
\frac{\partial G}{\partial t}+\boldsymbol{U} \cdot \boldsymbol{\nabla} G=S_{L}|\boldsymbol{\nabla} G| .
$$

In the present case, the Navier-Stokes are not ex- ${ }_{403}$ plicitly resolved and the velocity field is set as fol- 404 lows. First, $U^{u}$ and $V^{u}$, i.e. the velocity component 405 in the streamwise $x$ and transverse $y$ direction of un- 406 perturbed flow (before the generation of the vortex) 407 is given by $U^{u}(x, y)=-2 \int a(x) d x$ and $V^{u}(x, y)=408$ $a(x) \times y$, where $U_{0}=1.23 \mathrm{~m} \cdot \mathrm{s}^{-1}$ is the inlet velocity 409 of the burner and $a(x)=\partial V^{u} / \partial y(y=0)$ is the trans- 410 verse strain of the unperturbed flow. $a(x)$ was fitted 411 from experiments using a second order polynomial. 412 The coefficients of the polynomial were adjusted for 413 each equivalence ratio.

Secondly, the vortex velocity field was added to $U^{u} 415$ and $V^{u}$ and set using the Oseen expression. The in- 416 put parameters for the Oseen vortex are $U_{\theta}, X_{c}$ (the 417 streamwize location of the vortex center) and $R_{v}$, the 418 core-to-core distance. In the present case, by analyz- 419 ing experimental data (see Fig. 6), it was found that 420 $U_{\theta}$ does not vary with time and was therefore set to 421 a constant. The vortex center $X_{c}$ was convected at a ${ }_{422}$ velocity $U_{c}$, viz. $\partial X_{c} / \partial t=U_{c}$, where $U_{c}-U^{u}\left(X_{c}\right) \quad 423$

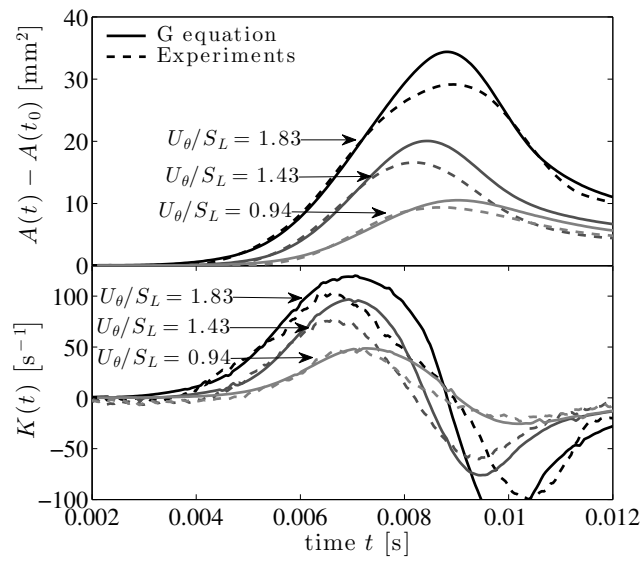

Figure 7: Time evolution of the flame area $A(t, \Delta)$ (a) and stretch $K(t, \Delta)$ (b) for $\Delta=10 \mathrm{~mm}$, for three different ratio of $U_{\theta} / S_{L}=0.94,1.43,1.83$. Dashed and full lines correspond respectively to experimental and numerical data

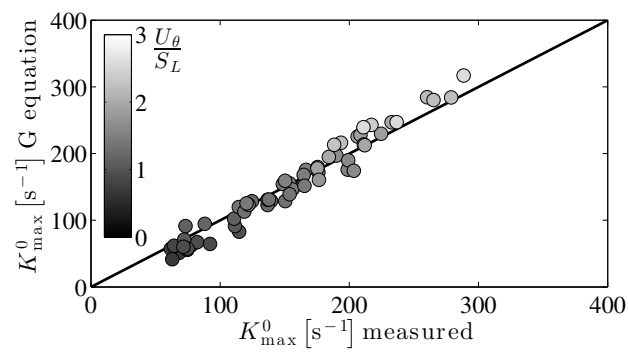

Figure 8: Maximum stretch $K_{\max }^{0}$ assessed by experiments versus $K_{\max }^{0}$ inferred from numerical simulations. Symbols are coloured by $U_{\theta} / S_{L}$

was found to be constant (see Fig. 6). The time evolution of the vortex ring diameter $R_{v}$ follows the relation [16] $R_{v}^{-1} \partial R_{v} / \partial t=a\left(X_{c}\right)$. In Fig. 6 the time evolution of vortex parameters issued from the experiments are compared to that prescribed in the numerical simulations. All quantities compare extremely well and thus validate the procedure for establishing the velocity field.

The $G$-field was initialized as a signed distance with the iso-value $G=G_{0}=0$ located at the streamwize location $x$ at which $U^{u}(x)=S_{L}$. Equation (3) is resolved using a fifth-order WENO discretization scheme in space and 4th-order RungeKutta scheme for time advancement. The usual reinitialization procedure is also applied at each time step so that the $G$-field remains a signed distance. The mesh size is $500 \times 500$ corresponding to a domain size of $25 \times 25 \mathrm{~mm}^{2}$. It was checked that increasing the mesh size up to $1000 \times 1000$ points yielded only marginal differences.

Numerical simulations have been validated against experimental data. Results for three different values 


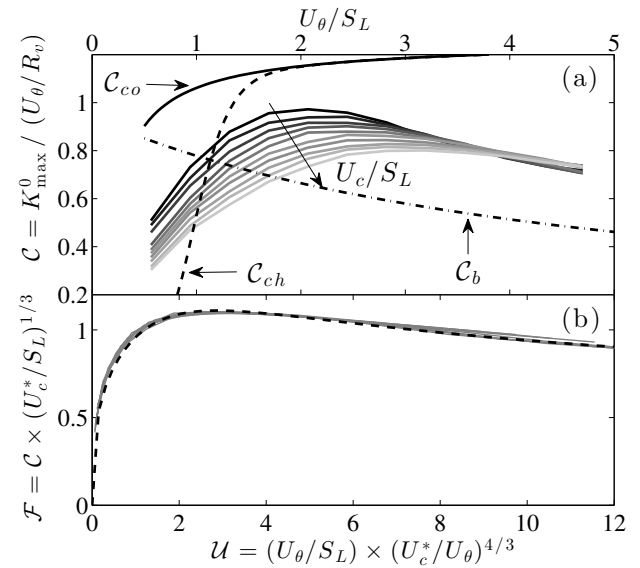

Figure 9: (a) Efficiency function as a function of $U_{\theta} / S_{L}$ for different values of $U_{c} / S_{L}$. The arrow indicates increasing values of $U_{c} / S_{L}$. (b) Rescaled efficiency function as a function of a rescaled velocity ratio. The black dashed line represent the fit using Eq. 4

of $U_{\theta} / S_{L}=0.94,1.43,1.83$ are presented in Fig. 7

The increase of $A(t, \Delta)$ is very nicely reproduced by the simulation, whilst some slight departures are observed close to the maximum of $A(t, \Delta)$. This indicates that the early stage of the interaction (i.e. before the vortex reaches the burnt gas) relies mainly on a kinematic interaction and that the heat release does not play a significant role at this stage. The simulated flame stretch compares favourably well with experiments for the three cases represented in Fig. 7] A scatter plot between the measured and simulated $K_{\max }^{0}$ for the entire database is further given in Fig. 8 Here again, a nice agreement is observed. Departures between numerical and experimental data for $K_{\max }^{0}$ lies within $20 \%$ on average.

\subsection{Formulation of a new efficiency function}

By use of such numerical simulations, the effect of $U_{c}$ and $U_{\theta}$ on $K_{\max }^{0}$ can thus be studied independently with the aim of incorporating these parameters in a more complete expression for the efficiency function.

In Fig. 9 a), are provided the numerical results for the efficiency function as a function of $U_{\theta} / S_{L}$ for $0.6 S_{L} \leq U_{c}-U^{u}\left(X_{c}\right) \leq 4.7 S_{L}$. Noticeable is the non monotonic evolution of $C$ with respect to $U_{\theta} / S_{L}$ that was previously observed in the experiments (see Fig. 47. Furthermore, one clearly sees a dependence of $C$ on $U_{c}$. Note that for $U_{\theta} / S_{L}>3.5$ the effect of $U_{c}$ is 482 almost negligible. In Fig. 9 b), it is shown that the ${ }_{483}$ evolution of $C$ with respect to $U_{c}$ and $U_{\theta}$ can collapse ${ }_{484}$ on a single curve, when the rescaled efficiency func- 485 tion $\mathcal{F}=C \times\left(U_{c}^{*} / S_{L}\right)^{1 / 3}$ is plotted as a function of ${ }_{486}$ a rescaled velocity ratio $\mathcal{U}=\left(U_{\theta} / S_{L}\right) \times\left(U_{c}^{*} / U_{\theta}\right)^{4 / 3}, 487$ where $U_{c}^{*}=U_{c}-U^{u}\left(X_{c}\right)+S_{L}$ is the relative ve- 488 locity between the flame and the vortex centers [4]. 489 This curve highlights a first zone for $\mathcal{U}<2.5$ where 490

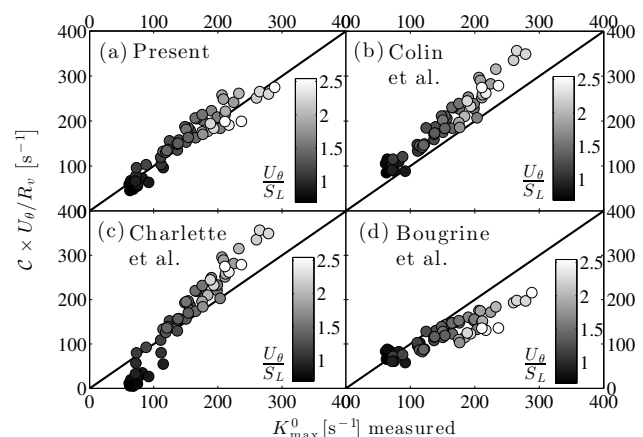

Figure 10: Scatter plot of the experimental vs modelled flame stretch using the present efficiency function (a), that of Colin et al. [7] (b), Charlette et al. [8] (c) and Bougrine et al. [9] (d). Symbols are coloured by $U_{\theta} / S_{L}$

$\mathcal{F}$ scales as $\mathcal{U}^{1 / 3}$, and a second zone at larger $\mathcal{U}$ for which $\mathcal{F}$ decreases as $\mathcal{U}^{-1 / 4}$. This trend can be well fitted by the following parametric expression (the black dashed line in Fig. 9(b))

$$
\mathcal{F}=\mathcal{U}^{1 / 3}\left[1+\left(\frac{\mathcal{U}}{\mathcal{U}_{\max }}\right)^{2}\right]^{\frac{-7}{24}},
$$

from which $C=\mathcal{F} \times\left(U_{c}^{*} / S_{L}\right)^{-1 / 3}$ can be recovered. $\mathcal{U}_{\max }$ is the rescaled velocity ratio for which the bending of $\mathcal{F}$ is observed and was found to be equal to 2.5 . The ability of this expression for modelling the flame stretch from the vortex strain is emphasized in Fig. 10 Departures between modelled and measured $K_{\max }^{0}$ are similar to Fig. 8, i.e. within $20 \%$. The present formulation for $C$ further appears to be more adequate than either that of Colin et al. [7], Charlette et al. [8] or Bougrine et al. [9].

\section{Conclusion}

The present study is devoted to the exploration of the flame stretch induced by a vortex dipole with special emphasis on the strain-sweeping competition. Both experiments and numerical simulations of a stagnation flame have been carried out, with the aim of assessing the ability of available parametric expression for describing the efficiency function. The outcomes of the present study can be summarized as follows:

- It was first shown that, though based on the same numerical data, $C$ provided by both Colin et al. [7], Charlette et al. [8] predict an increase of $C$ with respect to $U_{\theta} / S_{L}$ whereas that of Bougrine et al. [9] emphasizes the opposite trend. In addition, all these expressions fail in describing the non-monotonic evolution of $C$ with respect to $U_{\theta} / S_{L}$ which is observed in both experimental and numerical data. 
- Secondly, by comparing experiments to simpli- 548 fied numerical simulations based on the 'isother- 549 mal' G-equation, it was shown that the early 550 stage of interaction is driven by a kinematic in- 551 teraction between the vortex and the flame. The 552 maximum flame stretch issued from such numerical simulations is in agreement with experiments.

- Finally, these simulations allow the effect of the residence time of the vortex in the vicinity of the flame to be investigated. A new parametric ex- 556 pression for the efficiency function is proposed 557 and compares favourably well with experimen- 558 tal data.

As mentioned in the introduction, strain and sweep- ${ }^{560}$ ing effects are respectively representative of rather 562 small $\left(u_{r}\right)$ and large scales $\left(u^{\prime}\right)$ phenomena. This in- 563 dicates $a$ priori that in a LES, $C$ can be evaluated, 564 using the sub-grid scale velocity for $U_{\theta}$ and the to- ${ }^{565}$ tal (resolved + sub-grid scale) velocity for $U_{c}$. The ${ }^{566}$ residence time also requires the knowledge of the vor- ${ }^{567}$ tex sweeping direction compared to the flame normal ${ }_{569} 58$ direction, for which a sub-model has yet been devel- ${ }_{570}^{569}$ oped. Another important point concerns the fact that 571 in LES, the efficiency functions have to be integrated 572 over all the sub-grid scales. In previous studies, e.g. 573 [8], the integration was done in spectral space. It is 574 worth stressing that the integration over available tur- 575 bulent scales might not be necessary by keeping the ${ }^{576}$ description in physical space (that of the structure or ${ }^{577}$ correlation functions), in which the notion of cumula- ${ }_{578}^{578}$ tive over turbulent scales is implicit (see e.g. [17]). In- 580 deed, in Ref. [17], use was made of an expression for 581 the turbulent strain (Eq. (1) in [17]) which represents 582 the strain due to the combined effect of all smaller 583 scales (as the structure function does, see e.g. [18] 584 p.11, or [19] p 366). This expression is thus equiv- ${ }^{585}$ alent to the subgrid scale strain. Consequently, the ${ }^{586}$ multiplication of the efficiency function by the latter ${ }^{587}$ expression for the strain directly represents the flame ${ }_{589}^{58}$ stretch of all smaller scales than the scale considered. ${ }_{590}^{59}$ i.e. the subgrid flame stretch and there is no need for 591 spectral integration.

Further work will be devoted to exploring the impact of the ratio $R / \delta_{L}$ on $C$ by changing the diameter of the vortex generator. We also plan to study in detail the effect of fuel composition and especially the Lewis number effects.

\section{Acknowledgements}

The financial support from the Agence National de la Recherche under the project IDYLLE is gratefully acknowledged. In this respect, we would like to thank especially S. Richard, L. Selle and T. Poinsot for fruitful discussions. We are also thankful to the CNRS, the University of Orléans, and the French Government Program "Investissements d'avenir" through the LABEX CAPRYSSES. FT acknowledges EADS for its financial support. We also benefited from the computing resources provided by CaSciModOT. We thank Laurent Catherine for his technical assistance. We are grateful to Seong Young Lee for providing us the CHEMKIN PRO results.

\section{References}

[1] P.-H. Renard, D. Thevenin, J.-C. Rolon, S. Candel, Prog. Energ. Combust. 26 (2000) 225-282.

[2] T. Poinsot, D. Veynante, S. Candel, J. Fluid Mech. 228 (1991) 561-606

[3] W. L. Roberts, J. F. Driscoll, Combust. Flame 87 (1991) 245-256.

[4] M.-S. Wu, J. F. Driscoll, Combust. Flame 91 (1992) 310-322.

[5] W. L. Roberts, J. F. Driscoll, M. C. Drake, L. P. Goss, Combust. Flame 94 (1993) 58-69.

[6] T.-W. Lee, D. Santavicca, Combust. Sci. Technol. 90 (1993) 211-229.

[7] O. Colin, F. Ducros, D. Veynante, T. Poinsot, Phys. Fluids 12 (2000) 1843-1863.

[8] F. Charlette, C. Meneveau, D. Veynante, Combust. Flame 131 (2002) 159-180.

[9] S. Bougrine, S. Richard, O. Colin, D. Veynante, Flow, turbul. combust. 93 (2014) 259-281.

[10] J. F. Driscoll, Prog. Energ. Combust. 34 (2008) $91-$ 134

[11] A. Kolmogorov, Proc. USSR Ac. of Sci. 30 (1941) 299-303.

[12] H. Tennekes, J. Fluid Mech. 67 (1975) 561-567.

[13] C. Poulain, N. Mazellier, L. Chevillard, Y. Gagne, C. Baudet, Eur. Phys. J. B 53 (2006) 219-224.

[14] N. Bouvet, D. Davidenko, C. Chauveau, L. Pillier, Y. Yoon, Combust. Flame 161 (2014) 438-452.

[15] W. Thielicke, E. J. Stamhuis, Journal of Open Research Software 2 (2014) 30.

[16] R. Trieling, J. Van Wesenbeeck, G. Van Heijst, Phys. Fluids 10 (1998) 144-159.

[17] F. Thiesset, G. Maurice, F. Halter, N. Mazellier, C. Chauveau, I. Gökalp, Combustion Theory and Modelling 20 (2016) 1-17.

[18] A. A. R. Townsend, The structure of turbulent shear flow, Cambridge Univ Press, 1976.

[19] P. A. Davidson, Turbulence: an introduction for scientists and engineers, Oxford University Press, 2015. 